Proc. Estonian Acad. Sci. Phys. Math., 2007, 56, 1, 3-16

\title{
Galbed algebras and their sectional representation
}

\author{
Mart Abel and Veiko Lehto \\ Institute of Pure Mathematics, University of Tartu, J. Liivi 2, 51004 Tartu, Estonia; \\ mart.abel@ut.ee, veikolehto@hot.ee \\ Received 11 September 2006

\begin{abstract}
In this paper we prove that the unitization $A \times \mathbb{K}$ of a topological algebra $A$ is $\left(\alpha_{n}\right)$-galbed if and only if $A$ is $\left(\alpha_{n}\right)$-galbed. We also find sufficient conditions under which a unital strongly galbed algebra can be represented as a subalgebra of some section algebra.
\end{abstract}

Key words: galbed algebras, sectional representation, representations of topological algebras.

\section{INTRODUCTION}

Let $\mathbb{C}$ be the field of complex numbers, $\mathbb{R}$ the field of real numbers, $\mathbb{K}$ either $\mathbb{C}$ or $\mathbb{R}$, and $\mathbb{N}=\{0,1,2, \ldots\}$. From the definition of a topological vector space $E$ it follows that for each neighbourhood $O$ of zero in $E$, a fixed $n \in \mathbb{N}$ and fixed numbers $\lambda_{0}, \ldots, \lambda_{n} \in \mathbb{R}$ there exists a neigbourhood $U$ of zero in $E$ such that

$$
\sum_{k=0}^{n} \lambda_{k} U \subset O .
$$

By a topological algebra we mean a topological vector space $A$ over $\mathbb{K}$, which is also an associative algebra over $\mathbb{K}$ such that the multiplication in $A$ is separately continuous ${ }^{1}$. As usual, $l^{0}$ denotes the set of all sequences $\left(\alpha_{n}\right)$ of elements of $\mathbb{K}$, in which there is only a finite number of elements which are different from zero. By $l^{1}$ we denote the set of all sequences $\left(\alpha_{n}\right)$ of elements of $\mathbb{K}$ for which the series

$$
\sum_{k=0}^{\infty}\left|\alpha_{k}\right|
$$

1 It means that for every $a \in A$ and every neighbourhood $O$ of zero in $A$ there exists a neighbourhood $U$ of zero in $A$ such that $a U, U a \subset O$. 
converges. Let $l:=l^{1} \backslash l^{0}$. A topological algebra (or a topological vector space) $A$ is called a galbed algebra (a galbed space) if there exists a sequence $\left(\alpha_{n}\right) \in l$ such that for every neighbourhood $O$ of zero in $A$ there exists a neighbourhood $U$ of zero in $A$ such that

$$
\left\{\sum_{k=0}^{n} \alpha_{k} a_{k}: a_{0}, \ldots, a_{n} \in U\right\} \subset O
$$

for each $n \in \mathbb{N}$. If we have already specified such $\left(\alpha_{n}\right) \in l$ which makes $A$ a galbed algebra (a galbed space), then we call $A$ an $\left(\alpha_{n}\right)$-galbed algebra (an $\left(\alpha_{n}\right)$-galbed space). Moreover, if $\alpha_{0} \neq 0$ and

$$
\alpha:=\inf _{n>0}\left|\alpha_{n}\right|^{1 / n}>0,
$$

then $A$ is called a strongly galbed algebra (a strongly galbed space). A well-known example of a strongly galbed algebra (space) is an exponentially galbed algebra (an exponentially galbed space) $A$ in which for every neighbourhood $O$ of zero in $A$ there exists a neighbourhood $U$ of zero in $A$ such that

$$
\left\{\sum_{k=0}^{n} \frac{a_{k}}{2^{k}}: a_{0}, \ldots, a_{n} \in U\right\} \subset O
$$

for each $n \in \mathbb{N}$.

The terms "galbed space", "exponentially galbed space", and "exponentially galbed algebra" were introduced by Turpin (see $\left.\left[{ }^{1-5}\right]\right)$. The terms "galbed algebra" and "strongly galbed algebra" were introduced by Mati Abel and Mart Abel (see $\left[{ }^{6-12}\right]$ ). The present paper is based on Veiko Lehto's bachelor's thesis $\left[{ }^{13}\right]$. The need for writing this paper arises from the fact that $\left[{ }^{13}\right]$ was written in Estonian, but the results in $\left[{ }^{13}\right]$ might be also of interest as well as useful for readers, whose Estonian is not good enough to follow the proofs. In the present paper several results of Mart Abel and Mati Abel, known for exponentially galbed algebras, are generalized for the class of galbed algebras (in part 1) or for the class of strongly galbed algebras (in part 2).

\section{GALBED ALGEBRAS AND THEIR UNITIZATION}

\subsection{Strongly galbed algebras with bounded elements}

For every algebra $A$ let $Z(A)$ denote the centre of $A$. An element $a \in A$ is bounded (see $\left[{ }^{14}\right]$ ) in $A$ if there exists a number $\lambda \in \mathbb{C} \backslash\{0\}$ such that the set

$$
\left\{\left(\frac{a}{\lambda}\right)^{n}: n \in \mathbb{N}\right\}
$$

is bounded in $A$. 
Proposition 1. Let $\left(\alpha_{n}\right) \in l$ and I be a two-sided ideal in a topological algebra $A$. If $A$ is an $\left(\alpha_{n}\right)$-galbed algebra with bounded elements, then both $A / I$ and $Z(A / I)$ are $\left(\alpha_{n}\right)$-galbed algebras with bounded elements.

Proof. Let $\left(\alpha_{n}\right) \in l$ and $I$ be a two-sided ideal in a topological algebra $(A, \tau)$. Let $A$ be an $\left(\alpha_{n}\right)$-galbed algebra with bounded elements, $\pi: A \rightarrow A / I$ the canonical homomorphism, and $O^{\prime}$ a neighbourhood of zero in $\left(A / I, \tau_{I}\right)$, where $\tau_{I}$ stands for the quotient topology defined by the topology $\tau$ of $A$. Since the quotient map $\pi$ is continuous, $O=\pi^{-1}\left(O^{\prime}\right)$ is a neighbourhood of zero in $(A, \tau)$.

Since $A$ is $\left(\alpha_{n}\right)$-galbed, there exists a neighbourhood $U$ of zero in $A$ such that

$$
\left\{\sum_{k=0}^{n} \alpha_{k} a_{k}: a_{0}, \ldots, a_{n} \in U\right\} \subset O
$$

for each $n \in \mathbb{N}$. Take $V^{\prime}=\pi(U), n \in \mathbb{N}$ and arbitrary elements $x_{0}, \ldots, x_{n} \in V^{\prime}$. Then there exist elements $a_{0}, \ldots, a_{n} \in U$ such that $x_{i}=\pi\left(a_{i}\right)$ for every $i \in\{0, \ldots, n\}$. Notice that

$$
\sum_{k=0}^{n} \alpha_{k} x_{k}=\sum_{k=0}^{n} \alpha_{k} \pi\left(a_{k}\right)=\pi\left(\sum_{k=0}^{n} \alpha_{k} a_{k}\right) .
$$

It is clear that

$$
\pi\left(\left\{\sum_{k=0}^{n} \alpha_{k} a_{k}: a_{0}, \ldots, a_{n} \in U\right\}\right) \subset \pi(O)=\pi\left(\pi^{-1}\left(O^{\prime}\right)\right)=O^{\prime}
$$

for every $n \in \mathbb{N}$. Hence,

$$
\left\{\sum_{k=0}^{n} \alpha_{k} x_{k}: x_{0}, \ldots, x_{n} \in V^{\prime}\right\}=\pi\left(\left\{\sum_{k=0}^{n} \alpha_{k} a_{k}: a_{0}, \ldots, a_{n} \in U\right\}\right) \subset O^{\prime}
$$

for every $n \in \mathbb{N}$. Hence, $A / I$ is an $\left(\alpha_{n}\right)$-galbed algebra.

Let $O^{\prime \prime}$ be any neighbourhood of zero in $\left(Z(A / I), \tau_{Z}\right)$, where $\tau_{Z}=$ $\left\{T^{\prime} \cap Z(A / I): T^{\prime} \in \tau_{I}\right\}$ is the subspace topology on $Z(A / I)$, generated by $\tau_{I}$. Then there exists a neighbourhood $O^{\prime}$ of zero in $A / I$ such that $O^{\prime \prime}=O^{\prime} \cap Z(A / I)$. Since $A / I$ is $\left(\alpha_{n}\right)$-galbed, there exists a neigbourhood $V^{\prime}$ of zero in $A / I$ such that

$$
\left\{\sum_{k=0}^{n} \alpha_{k} x_{k}: x_{0}, \ldots, x_{n} \in V^{\prime}\right\} \subset O^{\prime}
$$

for every $n \in \mathbb{N}$. Fix an arbitrary $n \in \mathbb{N}$. Take $V^{\prime \prime}=V^{\prime} \cap Z(A / I)$ and arbitrary elements $y_{0}, \ldots, y_{n} \in V^{\prime \prime}$. Then 


$$
\sum_{k=0}^{n} \alpha_{k} y_{k} \in Z(A / I)
$$

and

$$
\left\{\sum_{k=0}^{n} \alpha_{k} y_{k}: y_{0}, \ldots, y_{n} \in V^{\prime \prime}\right\} \subset\left\{\sum_{k=0}^{n} \alpha_{k} x_{k}: x_{0}, \ldots, x_{n} \in V^{\prime}\right\} \subset O^{\prime} .
$$

Since $n \in \mathbb{N}$ was arbitrary, we get

$$
\left\{\sum_{k=0}^{n} \alpha_{k} y_{k}: y_{0}, \ldots, y_{n} \in V^{\prime \prime}\right\} \subset O^{\prime} \cap Z(A / I)=O^{\prime \prime}
$$

for every $n \in \mathbb{N}$. Hence, $Z(A / I)$ is an $\left(\alpha_{n}\right)$-galbed algebra.

Analogously to the second part of the proof of Theorem 2.1 in $\left[{ }^{6}\right]$, we get that all elements in $A / I$ and in $Z(A / I)$ are bounded.

Proposition 2. Let $A$ be a topological algebra and $\left(\alpha_{n}\right) \in l$. The unitization $A \times \mathbb{K}$ of $A$ in the product topology is an $\left(\alpha_{n}\right)$-galbed algebra if and only if $A$ is an $\left(\alpha_{n}\right)$-galbed algebra.

Proof. Let $\left(\alpha_{n}\right) \in l, A$ be a topological algebra and $O$ a neighbourhood of zero in $A \times \mathbb{K}$. Then there exist neighbourhoods of zero $U_{1}$ in $A$ and $V_{1}$ in $\mathbb{K}$ such that $U_{1} \times V_{1} \subset O$. If $A$ is $\left(\alpha_{n}\right)$-galbed, then there exist a neighbourhood $U_{2}$ of zero in $A$ and a number $\epsilon>0$ such that

$$
\left\{\sum_{k=0}^{n} \alpha_{k} a_{k}: a_{0}, \ldots, a_{n} \in U_{2}\right\} \subset U_{1}
$$

for every $n \in \mathbb{N}$ and $V_{2}=\{\lambda \in \mathbb{K}:|\lambda|<\epsilon\} \subset V_{1}$. Since $\left(\alpha_{n}\right) \in l$, we have

$$
0 \leq \sum_{k=0}^{n}\left|\alpha_{k}\right|<\sum_{k=0}^{\infty}\left|\alpha_{k}\right|<\infty
$$

for every $n \in \mathbb{N}$. Let

$$
\beta:=\sum_{k=0}^{\infty}\left|\alpha_{k}\right| \quad \text { and } \quad V_{3}:=\frac{1}{\beta} V_{2} .
$$

Now, $U_{2} \times V_{3}$ is a neighbourhood of zero in $A \times \mathbb{K}$. Fix an arbitrary $n \in \mathbb{N}$ and elements $\left(\alpha_{0}, \lambda_{0}\right), \ldots,\left(\alpha_{n}, \lambda_{n}\right) \in U_{2} \times V_{3}$. We have

$$
\sum_{k=0}^{n} \alpha_{k} a_{k} \in U_{1}
$$

6 
and

$$
\left|\sum_{k=0}^{n} \alpha_{k} \lambda_{k}\right| \leq \sum_{k=0}^{n}\left|\alpha_{k} \lambda_{k}\right| \leq \max _{i \in\{0, \ldots, n\}}\left|\lambda_{i}\right| \sum_{k=0}^{n}\left|\alpha_{k}\right|<\frac{\epsilon}{\beta} \beta=\epsilon .
$$

Hence,

$$
\sum_{k=0}^{n} \alpha_{k}\left(a_{k}, \lambda_{k}\right)=\left(\sum_{k=0}^{n} \alpha_{k} a_{k}, \sum_{k=0}^{n} \alpha_{k} \lambda_{k}\right) \in U_{1} \times V_{2} \subset O .
$$

Since $n \in \mathbb{N}$ was arbitrary,

$$
\left\{\sum_{k=0}^{n} \alpha_{k}\left(a_{k}, \lambda_{k}\right):\left(a_{0}, \lambda_{0}\right), \ldots,\left(a_{n}, \lambda_{n}\right) \in U_{2} \times V_{3}\right\} \subset O
$$

for every $n \in \mathbb{N}$, which means that $A \times \mathbb{K}$ is an $\left(\alpha_{n}\right)$-galbed algebra.

Conversely, let $A \times \mathbb{K}$ be an $\left(\alpha_{n}\right)$-galbed algebra for some $\left(\alpha_{n}\right) \in l$. Let $O$ be a neighbourhood of zero in $A$. Take any neighbourhood $V$ of zero in $\mathbb{K}$. Then $O \times V$ is a neighbourhood of zero in $A \times \mathbb{K}$. Hence, there exists a neighbourhood $W$ of zero in $A \times \mathbb{K}$ such that

$$
\left\{\sum_{k=0}^{n} \alpha_{k}\left(a_{k}, \lambda_{k}\right):\left(a_{0}, \lambda_{0}\right), \ldots,\left(a_{n}, \lambda_{n}\right) \in W\right\} \subset O \times V
$$

for every $n \in \mathbb{N}$. Now, there exist a neighbourhood $U$ of zero in $A$ and a neighbourhood $V_{1}$ of zero in $\mathbb{K}$ such that $U \times V_{1} \subset W$. Fix $n \in \mathbb{N}$ and $\left(a_{0}, \lambda_{0}\right), \ldots,\left(a_{n}, \lambda_{n}\right) \in U \times V_{1}$. Then

$$
\left(\sum_{k=0}^{n} \alpha_{k} a_{k}, \sum_{k=0}^{n} \alpha_{k} \lambda_{k}\right)=\sum_{k=0}^{n} \alpha_{k}\left(a_{k}, \lambda_{k}\right) \in O \times V .
$$

Hence,

$$
\left\{\sum_{k=0}^{n} \alpha_{k} a_{k}: a_{0}, \ldots, a_{n} \in U\right\} \subset O
$$

for every $n \in \mathbb{N}$, which means that $A$ is an $\left(\alpha_{n}\right)$-galbed algebra.

Proposition 3. Let $A$ be a topological algebra and $\left(\alpha_{n}\right) \in l$ a sequence such that $\alpha_{k} \neq 0$ for each $k \in \mathbb{N}$. The unitization $A \times \mathbb{K}$ of $A$ in the product topology is an $\left(\alpha_{n}\right)$-galbed algebra with bounded elements if and only if $A$ is an $\left(\alpha_{n}\right)$-galbed algebra with bounded elements.

Proof. Let $\left(\alpha_{n}\right) \in l$ be a sequence such that $\alpha_{k} \neq 0$ for every $k \in \mathbb{N}$.

Let $A$ be an $\left(\alpha_{n}\right)$-galbed algebra with bounded elements. By Proposition 2, $A \times \mathbb{K}$ is also an $\left(\alpha_{n}\right)$-galbed algebra. Let $\left(a_{0}, \lambda_{0}\right)$ be an arbitrary element of $A \times \mathbb{K}$ and $W$ an arbitrary neighbourhood of zero in $A \times \mathbb{K}$. Then there exist a number 
$\mu_{W}>0$, a balanced neighbourhood $O$ of zero in $A \times \mathbb{K}$, a neighbourhood $U$ of zero in $A$, and a balanced neighbourhood $V$ of zero in $K$ such that ${ }^{2} e_{A \times \mathbb{K}} \subset \mu_{W} O$ and $U \times V \subset O \subset W$. As $A$ is $\left(\alpha_{n}\right)$-galbed, there exists a balanced neighbourhood $U_{1}$ of zero in $A$ such that

$$
\left\{\sum_{k=0}^{n} \alpha_{k} c_{k}: c_{0}, \ldots, c_{n} \in U_{1}\right\} \subset U
$$

for every $n \in \mathbb{N}$. Since all elements of $A$ are bounded in $A$, there exist numbers $\lambda_{a_{0}} \in \mathbb{C} \backslash\{0\}, \mu_{1} \geq 1$, and $\gamma>0$ such that

$$
\left\{\left(\frac{a_{0}}{\lambda_{a_{0}}}\right)^{n}: n \in \mathbb{N}\right\} \subset \mu_{1} U_{1} \quad \text { and } \quad\{\lambda:|\lambda| \leq \gamma\} \subset V .
$$

Fix arbitrary $m \in \mathbb{N} \backslash\{0\}$ and put

$$
\begin{gathered}
\beta:=\min \left\{\min _{k \in\{1, \ldots, m\}}\left\{\left|\alpha_{k}\right|\right\}, \gamma\right\}, \\
\mu_{2}:=\max \left\{\left|\lambda_{a_{0}}\right|,\left|\lambda_{0}\right|\right\} \quad \text { and } \quad \mu_{0}:=\frac{2}{\sqrt[m]{\beta}} \mu_{2} .
\end{gathered}
$$

Let $k \in\{1, \ldots, m\} \subset \mathbb{N}$. Since $U_{1}$ is a balanced neighbourhood,

$$
\frac{1}{\alpha_{k}}\left(\begin{array}{c}
m \\
k
\end{array}\right)\left(\frac{a_{0}}{\mu_{0}}\right)^{k}\left(\frac{\lambda_{0}}{\mu_{0}}\right)^{m-k}=\frac{\left(\begin{array}{c}
m \\
k
\end{array}\right) \beta}{2^{m} \alpha_{k}}\left(\frac{\lambda_{a_{0}}}{\mu_{2}}\right)^{k}\left(\frac{\lambda_{0}}{\mu_{2}}\right)^{m-k}\left(\frac{a_{0}}{\lambda_{a_{0}}}\right)^{k},
$$

and

we get

$$
\left|\frac{\left(\begin{array}{c}
m \\
k
\end{array}\right) \beta}{2^{m} \alpha_{k}}\right|,\left|\left(\frac{\lambda_{a_{0}}}{\mu_{2}}\right)^{k}\right|,\left|\left(\frac{\lambda_{0}}{\mu_{2}}\right)^{m-k}\right| \leq 1
$$

$$
\frac{1}{\alpha_{k}}\left(\begin{array}{c}
m \\
k
\end{array}\right)\left(\frac{a_{0}}{\mu_{0}}\right)^{k}\left(\frac{\lambda_{0}}{\mu_{0}}\right)^{m-k} \in \mu_{1} U_{1}
$$

for each $k \in\{1, \ldots, m\}$. Therefore,

$$
\sum_{k=1}^{m} \alpha_{k}\left(\frac{1}{\alpha_{k}}\left(\begin{array}{c}
m \\
k
\end{array}\right)\left(\frac{a_{0}}{\mu_{0}}\right)^{k}\left(\frac{\lambda_{0}}{\mu_{0}}\right)^{m-k}\right)=\sum_{k=1}^{m}\left(\begin{array}{c}
m \\
k
\end{array}\right)\left(\frac{a_{0}}{\mu_{0}}\right)^{k}\left(\frac{\lambda_{0}}{\mu_{0}}\right)^{m-k} \in \mu_{1} U .
$$

Notice that

$$
\left|\left(\frac{\lambda_{0}}{\mu_{0}}\right)^{m}\right|=\left|\left(\frac{\lambda_{0}}{\mu_{2}}\right)^{m} \frac{\beta}{2^{m}}\right|=\left|\left(\frac{\lambda_{0}}{\mu_{2}}\right)^{m}\right| \frac{\beta}{2^{m}} \mid \leq 1 \cdot \frac{\gamma}{2^{m}}<\gamma .
$$

${ }^{2}$ Here $e_{A \times \mathbb{K}}=\left(\theta_{A}, 1\right)$ is the unit element of $A \times \mathbb{K}$. 
Since $V$ is balanced, we also have

$$
\left(\frac{\lambda_{0}}{\mu_{o}}\right)^{m} \in V=\mu_{1}\left(\frac{1}{\mu_{1}} V\right) \subset \mu_{1} V .
$$

Therefore,

$$
\left(\frac{\left(a_{0}, \lambda_{0}\right)}{\mu_{0}}\right)^{m}=\left(\sum_{k=1}^{m}\left(\begin{array}{c}
m \\
k
\end{array}\right)\left(\frac{a_{0}}{\mu_{0}}\right)^{k}\left(\frac{\lambda_{0}}{\mu_{0}}\right)^{m-k},\left(\frac{\lambda_{0}}{\mu_{0}}\right)^{m}\right) \in \mu_{1} O .
$$

Since $m \in \mathbb{N} \backslash\{0\}$ was arbitrary, we get

$$
\left(\frac{\left(a_{0}, \lambda_{0}\right)}{\mu_{0}}\right)^{m} \in \mu_{1} O
$$

for every $m \in \mathbb{N} \backslash\{0\}$. Remember that

$$
\left(\frac{\left(a_{0}, \lambda_{0}\right)}{\mu_{0}}\right)^{0}=e_{A \times \mathbb{K}} \subset \mu_{W} O .
$$

Take $\mu:=\max \left\{\mu_{1}, \mu_{W}\right\}$. Since $O$ is balanced,

$$
\left(\frac{\left(a_{0}, \lambda_{0}\right)}{\mu_{0}}\right)^{n} \in \mu O
$$

for every $n \in \mathbb{N}$, which means that all elements of $A \times \mathbb{K}$ are bounded.

Conversely, suppose that $A \times \mathbb{K}$ is an $\left(\alpha_{n}\right)$-galbed algebra with bounded elements. By Proposition 2, $A$ is also an $\left(\alpha_{n}\right)$-galbed algebra.

Let $a_{0}$ be an arbitrary element of $A$ and $U$ an arbitrary neighbourhood of zero in $A$. Fix any neighbourhood $V_{0}$ in $\mathbb{K}$. Then $U \times V_{0}$ is a neighbourhood of zero in $A \times \mathbb{K}$ and $\left(a_{0}, 0\right) \in A \times \mathbb{K}$. Since all elements of $A \times \mathbb{K}$ are bounded, there exist numbers $\lambda \in \mathbb{C} \backslash\{0\}$ and $\mu:=\mu_{U, V_{0}}>0$ such that

$$
\left\{\left(\left(\frac{a_{0}}{\lambda}\right)^{n}, 0\right): n \in \mathbb{N}\right\}=\left\{\left(\frac{\left(a_{0}, 0\right)}{\lambda}\right)^{n}: n \in \mathbb{N}\right\} \subset \mu\left(U \times V_{0}\right)=\mu U \times \mu V_{0} .
$$

Hence,

$$
\left\{\left(\frac{a_{0}}{\lambda_{0}}\right)^{n}: n \in \mathbb{N}\right\} \subset \mu U,
$$

which means that $a_{0}$ is bounded in $A$.

Corollary 1. Let $A$ be a topological algebra. The unitization $A \times \mathbb{K}$ of $A$ in the product topology is a strongly galbed algebra with bounded elements if and only if $A$ is a strongly galbed algebra with bounded elements. 
Proof. Let $A$ be a strongly galbed algebra with bounded elements. Then there exists a sequence $\left(\alpha_{n}\right) \in l$ with $\alpha_{0} \neq 0$ and

$$
\alpha:=\inf _{n>0}\left|\alpha_{n}\right|^{1 / n}>0
$$

such that $A$ is $\left(\alpha_{n}\right)$-galbed. Notice that $\alpha_{k} \neq 0$ for every $k \in \mathbb{N}$. Hence, $A \times \mathbb{K}$ is an $\left(\alpha_{n}\right)$-galbed algebra with bounded elements, by Proposition 3. Therefore, $A \times \mathbb{K}$ is also a strongly galbed algebra with bounded elements.

Conversely, let $A \times \mathbb{K}$ be a strongly galbed algebra with bounded elements. Then there exists a similar sequence $\left(\alpha_{n}\right) \in l$ as above. Notice that $\alpha_{k} \neq 0$ for every $k \in \mathbb{N}$. Hence, $A$ is an $\left(\alpha_{n}\right)$-galbed algebra with bounded elements, by Proposition 3. Therefore, $A$ is also a strongly galbed algebra with bounded elements.

\subsection{Galbed algebras with bounded elements}

Let $\left(\alpha_{n}\right) \in l$. Then there are infinitely many elements different from zero in $\left(\alpha_{n}\right)$. Let $P_{n}$ be the number of nonzero elements among $\alpha_{0}, \ldots, \alpha_{n}$. We will construct a sequence $\left(i_{n}\right)$ of indexes as follows:

$$
\begin{gathered}
i_{0}:=\min \left\{k: \alpha_{k} \neq 0\right\}, \\
i_{1}:=\min \left\{k: k>i_{0}, \alpha_{k} \neq 0\right\}, \\
i_{2}:=\min \left\{k: k>i_{1}, \alpha_{k} \neq 0\right\}, \\
\cdots \\
i_{j}:=\min \left\{k: k>i_{j-1}, \alpha_{k} \neq 0\right\}
\end{gathered}
$$

for every $j \in \mathbb{N} \backslash\{0\}$. Now we construct the sequence $\left(\beta_{n}\right)$ by $\beta_{k}:=\alpha_{i_{k}}$ for every $k \in \mathbb{N}$. Then the sequence $\left(\beta_{n}\right)$ consists of all nonzero elements of $\left(\alpha_{n}\right)$ and for every $m \in \mathbb{N}$,

$$
\sum_{k=0}^{m} \alpha_{k} a_{k}=\sum_{k=0}^{P_{m}-1} \beta_{k} b_{k},
$$

where $b_{k}=a_{i_{k}}$ for every $k \in \mathbb{N}$.

Definition 1. We call the sequence $\left(\beta_{n}\right) \in l$, constructed from $\left(\alpha_{n}\right) \in l$ as above, the underlying sequence of $\left(\alpha_{n}\right)$.

Proposition 4. Let $A$ be a topological algebra, $\left(\alpha_{n}\right) \in l$, and $\left(\beta_{n}\right)$ be the underlying sequence of $\left(\alpha_{n}\right)$. Then $A$ is an $\left(\alpha_{n}\right)$-galbed algebra if and only if $A$ is a $\left(\beta_{n}\right)$-galbed algebra. 
Proof. Let $A$ be an $\left(\alpha_{n}\right)$-galbed algebra and $O$ a neighbourhood of zero in $A$. Then there exists a neighbourhood $U$ of zero in $A$ such that

$$
\left\{\sum_{k=0}^{n} \alpha_{k} a_{k}: a_{0}, \ldots, a_{n} \in U\right\} \subset O
$$

for every $n \in \mathbb{N}$. Let $\left(\beta_{n}\right)$ be the underlying sequence of $\left(\alpha_{n}\right)$. Define $P_{-1}:=0$ and a map $f: \mathbb{N} \rightarrow \mathbb{N}$ as follows: for every $k \in \mathbb{N}$ find $m=m(k) \in \mathbb{N}$ such that $P_{m}=k+1$ and $P_{m-1}=k$. Take $f(k):=m$ and $b_{k}:=a_{f(k)}$ for every $k \in \mathbb{N}$. Then

$$
\sum_{k=0}^{n} \beta_{k} b_{k}=\sum_{k=0}^{f(n)} \alpha_{k} a_{k}
$$

for every $n \in \mathbb{N}$. Fix an arbitrary $l \in \mathbb{N}$ and consider the sets

$$
H_{1}:=\left\{\sum_{k=0}^{f(l)} \alpha_{k} a_{k}: a_{0}, \ldots, a_{f(l)} \in U\right\}
$$

and

$$
H_{2}:=\left\{\sum_{k=0}^{l} \beta_{k} b_{k}: b_{0}, \ldots, b_{l} \in U\right\} .
$$

For every element of $\mathrm{H}_{2}$ we have

$$
\sum_{k=0}^{l} \beta_{k} b_{k}=\sum_{k=0}^{f(l)} \alpha_{k} a_{k} \in H_{1} .
$$

Hence, $H_{2} \subset H_{1}$. As $l \in \mathbb{N}$ was arbitrary, we have

$$
\left\{\sum_{k=0}^{n} \beta_{k} b_{k}: b_{0}, \ldots, b_{n} \in U\right\} \subset H_{1} \subset O
$$

for every $n \in \mathbb{N}$. Hence, $A$ is a $\left(\beta_{n}\right)$-galbed algebra.

Conversely, let $A$ be a $\left(\beta_{n}\right)$-galbed algebra, where $\left(\beta_{n}\right)$ is the underlying sequence of $\left(\alpha_{n}\right)$. Moreover, let $O$ be any neighbourhood of zero in $A$. Then there exists a neighbourhood $U$ of zero in $A$ such that

$$
\left\{\sum_{k=0}^{n} \beta_{k} b_{k}: b_{0}, \ldots, b_{n} \in U\right\} \subset O
$$

for each $n \in \mathbb{N}$. Fix an arbitrary $m \in \mathbb{N}$ and consider the sets

$$
G_{1}:=\left\{\sum_{k=0}^{m} \alpha_{k} a_{k}: a_{0}, \ldots, a_{m} \in U\right\},
$$




$$
G_{2}:=\left\{\sum_{k=0}^{P_{m}-1} \beta_{k} b_{k}: b_{0}, \ldots, b_{P_{m}-1} \in U\right\}
$$

with $\beta_{k}=\alpha_{i_{k}}$ as above. Take $a_{j}:=b_{k}$ for $j=i_{k}$ and $a_{j}:=\theta_{A}$ otherwise. Since for every element of $G_{1}$ we have

$$
\sum_{k=0}^{m} \alpha_{k} a_{k}=\sum_{k=0}^{P_{m}-1} \beta_{k} b_{k} \in G_{2},
$$

$G_{1} \subset G_{2}$. As $m \in \mathbb{N}$ is arbitrary, we have

$$
\left\{\sum_{k=0}^{n} \alpha_{k} a_{k}: a_{0}, \ldots, a_{n} \in U\right\} \subset G_{2} \subset O
$$

for each $n \in \mathbb{N}$, which means that $A$ is an $\left(\alpha_{n}\right)$-galbed algebra.

Next, we give two simple corollaries.

Corollary 2. Let $A$ be a topological algebra, $\left(\alpha_{n}\right) \in l$, and $\left(\beta_{n}\right)$ be the underlying sequence of $\left(\alpha_{n}\right)$. Then $A \times \mathbb{K}$ is an $\left(\alpha_{n}\right)$-galbed algebra if and only if $A \times \mathbb{K}$ is a $\left(\beta_{n}\right)$-galbed algebra.

Proof. The result follows immediately if we put $A \times \mathbb{K}$ instead of $A$ in Proposition 4 .

Corollary 3. Let $A$ be a topological algebra, $\left(\alpha_{n}\right) \in l$, and $\left(\beta_{n}\right)$ be the underlying sequence of $\left(\alpha_{n}\right)$. Then $A$ is a $\left(\beta_{n}\right)$-galbed algebra with bounded elements if and only if $A \times \mathbb{K}$ is a $\left(\beta_{n}\right)$-galbed algebra with bounded elements.

Proof. Since $\beta_{k} \neq 0$ for every $k \in \mathbb{N}$, the desired result follows directly if we substitute $\left(\beta_{n}\right)$ for $\left(\alpha_{n}\right)$ in Proposition 3.

Now we are ready for our first main result.

Theorem 1. Let $A$ be a topological algebra and $\left(\alpha_{n}\right) \in l$. Then $A$ is an $\left(\alpha_{n}\right)$ galbed algebra with bounded elements if and only if $A \times \mathbb{K}$ is an $\left(\alpha_{n}\right)$-galbed algebra with bounded elements.

Proof. Let $A$ be a topological algebra, $\left(\alpha_{n}\right) \in l$, and $\left(\beta_{n}\right)$ be the underlying sequence of $\left(\alpha_{n}\right)$. By Proposition 4, $A$ is an $\left(\alpha_{n}\right)$-galbed algebra (with bounded elements) if and only if $A$ is a $\left(\beta_{n}\right)$-galbed algebra (with bounded elements). By Corollary $3, A$ is a $\left(\beta_{n}\right)$-galbed algebra with bounded elements if and only if $A \times \mathbb{K}$ is a $\left(\beta_{n}\right)$-galbed algebra with bounded elements. By Corollary $2, A \times \mathbb{K}$ is a $\left(\beta_{n}\right)$-galbed algebra (with bounded elements) if and only if $A \times \mathbb{K}$ is an $\left(\alpha_{n}\right)$ galbed algebra (with bounded elements). Hence, $A$ is an $\left(\alpha_{n}\right)$-galbed algebra with bounded elements if and only if $A \times \mathbb{K}$ is an $\left(\alpha_{n}\right)$-galbed algebra with bounded elements. 


\section{SECTIONAL REPRESENTATION OF UNITAL STRONGLY GALBED ALGEBRAS}

In this section we generalize part c) of Theorem 3.13 in $\left[{ }^{6}\right]$ from exponentially galbed algebras to strongly galbed algebras. For this purpose we need to generalize some other results. Throughout this section, we consider only topological algebras over $\mathbb{C}$ (i.e., $\mathbb{K}=\mathbb{C}$ in the definition of a topological algebra). Remember that in unital algebras every ideal is regular.

A topological algebra $A$ is $\sigma$-complete if every Cauchy net of elements of $A$ converges in $A$. A topological algebra $A$ is topologically primitive if there exists a closed maximal regular left (or right) ideal $M$ of $A$ such that $\{a \in A: a A \subset M\}$ $=\left\{\theta_{A}\right\}\left(\{a \in A: A a \subset M\}=\left\{\theta_{A}\right\}\right.$, respectively). Let $M$ be a maximal regular left (or right) ideal of $A$. Then the two-sided ideal $P=\{a \in A: a A \in M\}$ ( $P=\{a \in A: A a \in M\}$, respectively) is a primitive ideal of $A$, defined by $M$.

Proposition 5. Let $A$ be a unital $\sigma$-complete topologically primitive strongly galbed Hausdorff algebra (over $\mathbb{C}$ ) with bounded elements and $P$ a primitive ideal, defined by a closed maximal left (or right) ideal of $A$. Then $Z(A / P)$ is topologically isomorphic to $\mathbb{C}$.

Proof. Since $P$ is a primitive ideal defined by a closed maximal left (or right) ideal of $A, P$ is closed. Now, $A / P$ is topologically primitive by $\left[{ }^{15}\right]$, Proposition 9 , and Hausdorff by $\left[{ }^{16}\right]$, Proposition 5 . As in the proof of Theorem 2 in $\left[{ }^{16}\right]$, it is easy to show that $A / P$ is $\sigma$-complete. As $A / P$ is strongly galbed algebra with bounded elements by Proposition $1, Z(A / P)$ is topologically isomorphic to $\mathbb{C}$ by Theorem 3.1 of $\left[{ }^{7}\right]$.

The set of all regular two-sided ideals of a topological algebra $A$, which are maximal as left or right ideals, is denoted by $m(A)$. Let now $A$ be a unital topological algebra and $B$ a closed subalgebra of $Z(A)$, containing the unital element $e_{A}$ of $A$. An ideal $M \in m(B)$ is an extendible ideal of $B$ if

$$
I(M):=\operatorname{cl}_{A}\left\{\sum_{k=1}^{n} a_{k} m_{k}: n \in \mathbb{N}, a_{1}, \ldots, a_{n} \in A, m_{1}, \ldots, m_{n} \in M\right\} \neq A .
$$

The set of all extendible ideals of $B$ is denoted by $m_{e}(B)$. For general definitions see $\left[{ }^{6}\right]$, pp. $18-19$, or $\left[{ }^{13}\right]$, pp. $4-5$.

Proposition 6. Let A be a unital $\sigma$-complete topologically primitive strongly galbed Hausdorff algebra with bounded elements, $M$ a closed maximal left (right or twosided) ideal of $A$, and $B$ a closed subalgebra of $Z(A)$, containing the unital element $e_{A}$ of $A$. Then

1) every $b \in B$ defines a number $\lambda \in \mathbb{C}$ such that $b-\lambda e_{a} \in M$;

2) $M \cap B \in m_{e}(B)$. 
Proof. The proof is similar to the proof of Proposition 3.1 in $\left[{ }^{6}\right]$, because we need only the fact that $A / P$ is topologically isomorphic to $\mathbb{C}$ for a primitive ideal $P$ defined by $M$. For detailed proof see the proof of Proposition 2.8 in $\left[{ }^{13}\right]$.

For every $M \in m_{e}(B)$ and $a \in A$ let $A_{M}:=A / I(M)$, let $\kappa_{M}: A \rightarrow A_{M}$ be the canonical homomorphism and $a^{\wedge}: m_{e}(B) \rightarrow A_{M}$ a map, defined by $a^{\wedge}(M):=$ $\kappa_{M}(a)$. Let $I$ be a closed maximal left (right or two-sided) ideal of $A$ and $J$ be a closed maximal left (right or two-sided) ideal of $A_{M}$. Then $\kappa_{M}(I)=\left\{a^{\wedge}(M)\right.$ : $a \in I\}$ and $\kappa_{M}^{-1}(J)=\left\{a \in A: a^{\wedge}(M) \in J\right\}$.

Proposition 7. Let $A$ be a unital $\sigma$-complete topologically primitive strongly galbed Hausdorff algebra with bounded elements, I a closed maximal left (right or two-sided) ideal of $A$, and $B$ a closed subalgebra of $Z(A)$, containing the unital element $e_{A}$ of $A$. Then there exists $M \in m_{e}(B)$ and a closed maximal left (right or two-sided, respectively) ideal $J$ of $A_{M}$ such that $I=\kappa_{M}^{-1}(J)$.

Proof. Let $I$ be a closed maximal left (right or two-sided) ideal of $A$. By Proposition 6, part 2), $M:=I \cap B \in m_{e}(B)$. Let $J:=\kappa_{M}(I)$. Then $J$ is a closed left (right or two-sided) ideal of $A_{M}$ and $\kappa_{M}^{-1}(J)$ is a closed maximal left (right or two-sided, respectively) ideal of $A$ by Lemma 3.5 of $\left[{ }^{6}\right]$, pp. 52-54. Moreover, $I \subset \kappa_{M}^{-1}(J)$. Since $I$ is maximal left (right or two-sided, respectively) ideal of $A, I=\kappa_{M}^{-1}(J)$.

The complex (or the triple) $(B, \pi, X)$, where $B$ and $X$ are topological spaces and $\pi: B \rightarrow X$ is a continuous open surjection, is called a fibre bundle. A map $f: X \rightarrow B$ is said to be a section of the fibre bundle $(B, \pi, X)$ or shortly, a section of $\pi$ if $\pi[f(x)]=x$ for all $x \in X$. The set of all continuous sections of $\pi$ is denoted by $\Gamma(\pi)$. If all the fibres $B_{x}:=\{b \in B: \pi(b)=x\}$ of the fibre bundle $(B, \pi, X)$ are topological algebras, it is possible to define algebraic operations and topology on $\Gamma(\pi)$ so that $\Gamma(\pi)$ becomes a topological algebra (see $\left[{ }^{6}\right]$, p. 22 , or $\left[{ }^{13}\right]$, p. 8 ). This topological algebra is called a section algebra.

Let $A$ and $B$ be topological algebras. Every continuous homomorphism $f: A \rightarrow B$ is a representation of $A$ in $B$. If $A$ is the section algebra $\Gamma(\pi)$ for some fibre bundle $(B, \pi, X)$, then $f$ is a sectional representation of $A$.

For every representation $f: A \rightarrow B$ we can consider $B$ as a left (or right) $A$-module if we define the module multiplication $\cdot_{f}$ in $B$ by $a \cdot{ }_{f} b:=f(a) b$ $\left(b \cdot f a:=f(a) b\right.$, respectively) for each $a \in A$ and $b \in B$. If $B$ and $\left\{\theta_{B}\right\}$ are the only $A$-submodules of $B$, we call $f$ an irreducible representation.

A topological algebra $A$ over $\mathbb{C}$ is a Gelfand-Mazur algebra if the quotient algebra $A / M$ is topologically isomorphic to $\mathbb{C}$ for every $M \in m(A)$. The intersection of the kernels of all irreducible representations of a topological algebra $A$ is called the topological radical of $A$ and is denoted by $\operatorname{rad} A$. It is known that if $A$ is a Gelfand-Mazur algebra, then $\operatorname{rad} A$ is the intersection of all closed maximal regular left (or right) ideals of $A$. Moreover, every strongly galbed algebra with bounded elements is a Gelfand-Mazur algebra by Theorem 4.2 of $\left[{ }^{12}\right]$. 
Proposition 8. Let $A$ be a unital $\sigma$-complete topologically primitive strongly galbed Hausdorff algebra with bounded elements, I a closed maximal left (right or two-sided) ideal of $A$, and $B$ a closed subalgebra of $Z(A)$, containing the unital element $e_{A}$ of $A$. Then

$$
\operatorname{rad} A=\bigcap\left\{\kappa_{M}^{-1}\left(\operatorname{rad} A_{M}\right): M \in m_{e}(B)\right\} .
$$

Proof. The proof is similar to the proof of Proposition 3.7 in $\left[{ }^{6}\right]$.

Let $A$ be a unital strongly galbed algebra (over $\mathbb{C}$ ) with bounded elements and $B$ a subalgebra of $Z(A)$, for which $m_{e}(B) \neq \emptyset$. As in Proposition 1, we can show that $B$ is a strongly galbed algebra with bounded elements, thus a Gelfand-Mazur algebra. Hence, for every $M \in m_{e}(B)$ there exists a nontrivial homomorphism $\phi_{M}: B \rightarrow \mathbb{C}$ such that $\operatorname{ker} \phi_{M}=M$. Let

$$
\Delta:=\bigcup_{M \in m_{e}(B)} A_{M} .
$$

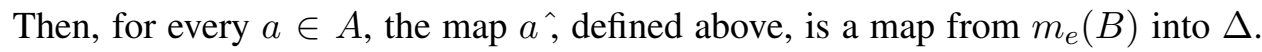
Let $\pi: \Delta \rightarrow m_{e}(B)$ be a map which assigns to every $d \in \Delta$ the ideal $M \in m_{e}(B)$ for which $d \in A_{M}$. In $\left[{ }^{6}\right]$, pp. 60-61, it is shown that such $\pi$ is a well-defined open and continuous map and that $A_{M_{1}} \cap A_{m_{2}} \neq \emptyset$ if and only if $M_{1}=M_{2}$.

On $m_{e}(B)$ we consider the Gelfand topology $\tau$; a subbase of neighbourhoods of $M_{0} \in m_{e}(B)$ consists of sets

$$
O\left(M_{0}, \epsilon, b\right):=\left\{M \in m_{e}(B): \mid\left(\phi_{M}-\phi_{M_{0}} \mid<\epsilon\right\},\right.
$$

where $\epsilon>0$ and $b \in B$ vary. On $A_{M}$ we consider the quotient topology $\tau_{M}$ and on $\Delta$ the topology $\tau_{\Delta}:=\left\{\pi^{-1}(U): U \in \tau\right\}$. Then $A_{M}$ is a topological algebra, $\left(\Delta, \pi, m_{e}(B)\right)$ is a fibre bundle, and $a^{\wedge} \in \Gamma(\pi)$ for every $a \in A$.

Let $\Upsilon: A \rightarrow \Gamma(\pi)$ be a map, defined by $\Upsilon(a):=a^{\wedge}$ for every $a \in A$. It is known (see $\left[{ }^{6}\right]$, pp. 60-61) that $\Upsilon$ is a continuous map and hence, a sectional representation of $A$.

Proposition 9. Let $A$ be a unital $\sigma$-complete topologically primitive topologically semisimple strongly galbed Hausdorff algebra with bounded elements. Then the map $\Upsilon$ is one-to-one.

Proof. Using Proposition 8, the proof becomes similar to the proof of Proposition 3.12 in $\left[{ }^{6}\right]$.

Finally, we give our second main result of this paper.

Theorem 2. Let $A$ be a unital $\sigma$-complete topologically primitive topologically semisimple strongly galbed Hausdorff algebra (over $\mathbb{C}$ ) with bounded elements. Then A can be considered as a subalgebra of the section algebra $\Gamma(\pi)$.

Proof. Since $\Upsilon$ is a one-to-one sectional representation by Proposition 9, $A$ can be considered as a subalgebra of $\Gamma(\pi)$. 


\section{REFERENCES}

1. Turpin, Ph. Généralisations d'un théorème de S. Mazur et W. Orlicz. C. R. Acad. Sci. Paris, 1971, 273, 457-460.

2. Turpin, Ph. Variantes de résultats de S. Mazur et W. Orlicz. C. R. Acad. Sci. Paris, 1971, 273, 506-509.

3. Turpin, Ph. Espaces et intersections d'espaces d'Orlicz non localement convexes. Studia Math., 1973, 46, 167-195.

4. Turpin, Ph. Espaces et opérateurs exponentiellement galbés. In Seminaire Pierre Lelong (Analyse) Année 1973/74 (Lelong, P., ed.), Lecture Notes in Math., 1975, 474, 48-62.

5. Turpin, Ph. Convexités dans les espaces vectoriels topologiques généraux. Dissertationes Math. (Rozprawy Mat.), 131, PWN, Warszawa, 1976.

6. Abel, Mart. Structure of Gelfand-Mazur Algebras. Dissertationes Mathematicae Universitatis Tartuensis, 31, Tartu University Press, Tartu, 2003.

7. Abel, Mart. The center of topologically primitive galbed algebras. In Topological Algebras, Their Applications and Related Topics (Jarosz, K. and Sołtysiak, A., eds), Banach Center Publ., 2005, 67, 45-54.

8. Abel, Mart. On the center of galbed algebras. Bull. Greek Math. Soc. (to appear).

9. Abel, Mart and Abel, Mati. The center of topologically primitive exponentially galbed algebras. Int. J. Math. Math. Sci., 2006, Art. ID 19697, 1-10.

10. Abel, Mart and Abel, Mati. On galbed algebras and galbed spaces (to appear).

11. Abel, Mati. On the Gelfand-Mazur theorem for exponentially galbed algebras. Tartu Ülik. Toimetised, 1990, 899, 65-70.

12. Abel, Mati. Galbed Gelfand-Mazur algebras. In Topological Algebras and Their Applications (Arizmendi, H., Bosch, C. and Palacios, L., eds), Contemp. Math., 2004, 341, $17-24$.

13. Lehto, V. Galbed Algebras and Their Sectional Representation. Bachelor's Thesis, Tartu, 2006 (in Estonian).

14. Allan, G. R. A spectral theory for locally convex algebras. Proc. London Math. Soc., 1965, 15, 399-421.

15. Bonsall, F. F. and Duncan, J. Complete normed algebras. Ergeb. Math. Grenzgeb., 1973, 80.

16. Horváth, J. Topological Vector Spaces and Distributions I. Addison-Wesley Publ. Co., Reading, Mass.-London- Don Mills, Ont., 1966.

\section{Gälbalgebrad ja nende lõikeesitus}

\section{Mart Abel ja Veiko Lehto}

On näidatud, et topoloogiline algebra $A$ on $\left(\alpha_{n}\right)$-gälb algebra siis ja ainult siis, kui algebrale $A$ ühiku juurdetoomisel tekkinud algebra on $\left(\alpha_{n}\right)$-gälb algebra. Teise põhitulemusena on leitud piisavad tingimused selleks, et ühikuga tugevalt gälb algebra oleks vaadeldav teatava lõikekujutuste algebra alamalgebrana. Artikli aluseks on töö $\left[{ }^{13}\right]$. 